\title{
High efficiency multilayer blazed gratings for EUV and soft X-rays: Recent developments
}

\author{
Dmitriy L. Voronov, ${ }^{\text {a }}$ Minseung Ahn, ${ }^{\mathrm{b}}$ Erik H. Anderson, ${ }^{\mathrm{a}}$ Rossana Cambie, ${ }^{\mathrm{a}}$ Chih-Hao Chang, \\ Leonid I. Goray, ${ }^{\mathrm{c}, \mathrm{d}}$ Eric M. Gullikson, ${ }^{\mathrm{a}}$ Ralf K. Heilmann, ${ }^{\mathrm{b}}$ Farhad Salmassi, ${ }^{\mathrm{a}}$ \\ Mark L. Schattenburg, ${ }^{\mathrm{b}}$ Tony Warwick, ${ }^{\mathrm{a}}$ Valeriy V. Yashchuk ${ }^{\mathrm{a}}$, and Howard A. Padmore ${ }^{\mathrm{a}}$ \\ ${ }^{\text {a }}$ Lawrence Berkeley National Laboratory, Berkeley, California, 94720 \\ ${ }^{\mathrm{b}}$ Massachusetts Institute of Technology, Cambridge, Massachusetts, 02139 \\ ${ }^{c}$ Saint Petersburg Academic University, Khlopina 8/3, St. Petersburg, 194021, Russia \\ ${ }^{\mathrm{d}}$ Institute for Analytical Instrumentation, Rizhsky Prospect 26, St. Petersburg, 190103, Russia
}

\begin{abstract}
Multilayer coated blazed gratings with high groove density are the best candidates for use in high resolution EUV and soft x-ray spectroscopy. Theoretical analysis shows that such a grating can be potentially optimized for high dispersion and spectral resolution in a desired high diffraction order without significant loss of diffraction efficiency. In order to realize this potential, the grating fabrication process should provide a perfect triangular groove profile and an extremely smooth surface of the blazed facets. Here we report on recent progress achieved at the Advanced Light Source (ALS) in fabrication of high quality multilayer coated blazed gratings. The blazed gratings were fabricated using scanning beam interference lithography followed by wet anisotropic etching of silicon. A $200 \mathrm{~nm}$ period grating coated with a Mo/Si multilayer composed with 30 bi-layers demonstrated an absolute efficiency of $37.6 \%$ in the $3^{\text {rd }}$ diffraction order at 13.6 $\mathrm{nm}$ wavelength. The groove profile of the grating was thoroughly characterized with atomic force microscopy before and after the multilayer deposition. The obtained metrology data were used for simulation of the grating efficiency with the vector electromagnetic PCGrate-6.1 code. The simulations showed that smoothing of the grating profile during the multilayer deposition is the main reason for efficiency losses compared to the theoretical maximum. Investigation of the grating with cross-sectional transmission electron microscopy revealed a complex evolution of the groove profile in the course of the multilayer deposition. Impact of the shadowing and smoothing processes on growth of the multilayer on the surface of the sawtooth substrate is discussed.
\end{abstract}

Key words: diffraction grating, diffraction efficiency, blazed grating, multilayer coating, scanning beam interference lithography, wet anisotropic etch, EUV, soft x-rays, TEM

\section{INTRODUCTION}

Recent progress in fabrication of high quality sawtooth substrates by wet anisotropic etch of silicon single crystals ${ }^{1}$ opens promising prospects for development of EUV and soft x-ray gratings having very high diffraction efficiency, dispersion, and spectral resolution. Such gratings would have a wide range of applications such as ultra high resolution soft x-ray spectroscopy, ${ }^{2}$ astrophysics, ${ }^{3}$ pulse compression of chirped x-ray beams, etc. There are however, several technological challenges which need to be overcome in order to approach the theoretical diffraction efficiency. The most important one is deposition of the multilayer coating, which provides high reflectance of the grating grooves. While multilayer deposition is a well established process for flat or substrates with small curvature, multilayer growth on the highly corrugated surface of the blazed gratings is complex and requires additional studies. In our previous work ${ }^{1}$ we achieved a reasonably high efficiency of $33 \%$ for a grating coated with $20 \mathrm{Mo} / \mathrm{Si}$ pairs, but it was significantly lower than the reflectance of $52 \%$ of the witness multilayer deposited on the flat substrate with comparable surface roughness. We found that the groove profile of the grating had undergone significant modification after the multilayer deposition. One can assume that this groove deformation has a considerable contribution to the reduction of the grating efficiency.

*dlvoronov@lbl.gov; phone 1510 486-4863; fax 15104952591 
Moreover, groove profile distortion is expected to increase with the total thickness of the multilayer coating. This constrains the deposition to a limited number of bi-layers, resulting in additional efficiency losses due to reduction of the multilayer reflectance. Indeed, a much thicker multilayer is required in order to realize the highest reflectance, which can reach $70 \%$ for multilayers composed of 50 pairs or more. ${ }^{4}$ All these issues however should be investigated experimentally and by simulation.

In this paper we report on fabrication and characterization of a $200 \mathrm{~nm}$ period EUV Mo/Si-coated blazed grating with a demonstrated $37.6 \%$ efficiency in the $3^{\text {rd }}$ diffraction order. We investigated the impact of the groove profile on multilayer blazed grating performance by simulation of the efficiency for the model gratings which have ideal and experimental profiles. We also performed detailed TEM examination of structure of the multilayer stack, which reveals evolution of the groove profile during the multilayer deposition.

\section{EXPERIMENT}

Sawtooth substrates with a period of $200 \mathrm{~nm}$ and a blaze angle of 6 degrees were fabricated by scanning beam interference lithography and anisotropic $\mathrm{KOH}$ etch of silicon single crystals as was described in detail earlier. ${ }^{1}$ Following an etch/oxidation procedure, silicon nubs from the initial lithography and ion etch were removed resulting in a near perfect triangular profile of the grating grooves. The procedure includes oxidation of the grating surface with Piranha solution followed by selective etch of the silicon oxide with hydrofluoric acid. In total 26 oxidation/oxide etch cycles were applied to the grating described in this paper. Thorough control of the etch parameters such as an etch temperature, solution concentration, purity of the chemicals and the sample surface, etc. provided high reproducibility of the fabrication process.

The sawtooth substrate with a dimension of $5 \times 15 \mathrm{~mm}^{2}$ was coated with a Mo/Si multilayer composed of 30 bi-layers by dc-magnetron sputtering at the Center for X-ray Optics at LBNL. ${ }^{15}$ The bi-layer d-spacing was targeted to $7.1 \mathrm{~nm}$ in order to bring the $3^{\text {rd }}$ diffraction order of the grating into the blaze condition. The deposition was performed by constantrate motion of the substrate, placed on a carousel, over an aperture designed to provide thickness uniformity of the coating for flat substrates. The distance between the target and the substrate was approximately equal to the diameter of the magnetron target erosion zone, so each point on the substrate collected the atomic flux from a wide spatial cone with an opening of approximately 50-60 degrees. Large flux divergence can cause a shadowing effect on the corrugated surface of the grating, especially in the vicinity of anti-blazed facets which have a slope angle of 64 degrees to the substrate surface. In order to mitigate the shadowing of the anti-blazed facets, the substrate was off-centered slightly with respect to the center of the magnetron as shown in Fig. 1 (the direction of the substrate motion is perpendicular to the plane of the figure). This resulted in about a 10-degree angle between the average direction of atomic flux towards the center of the grating and the normal to the grating surface.

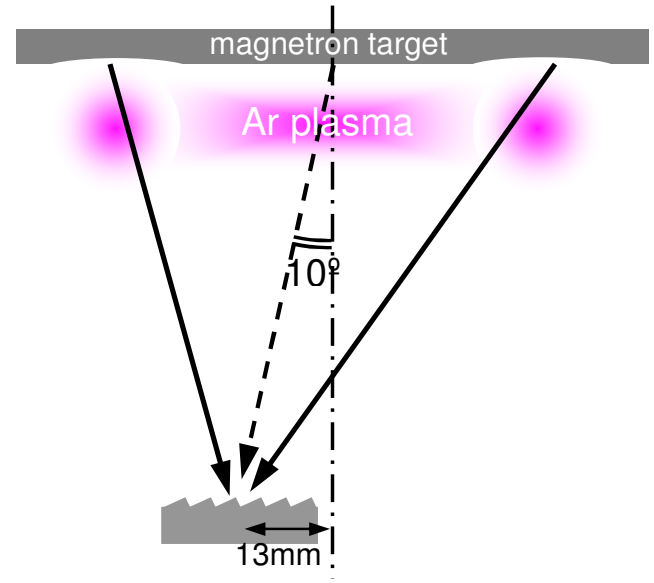

Figure 1. Position of the sawtooth substrate with respect to the magnetron target during the Mo/Si multilayer deposition. The center of the substrate was shifted by $13 \mathrm{~mm}$ to the left in order to reduce possible shadowing of the highly tilted anti-blazed facets. The direction of the substrate motion is perpendicular to the plane of the figure. 
The profile of the grating before and after multilayer deposition was measured by AFM. In order to obtain average groove profiles suitable for simulation, all 512 lines of $1 \times 1 \mu \mathrm{m}^{2}$ scans were averaged with an averaging option of the AFM Nanospec software. The averaged profiles were used for the simulation of absolute diffraction efficiency of multilayer coated gratings using PCGrate 6.1 software based on the rigorous approach of boundary integral equations. ${ }^{6}$

Diffraction efficiency of the multilayer coated grating was measured at the beamline 6.3.2, at the ALS. ${ }^{7}$ The incidence angle was set to $11^{\circ}$ with respect to the grating normal, which corresponded to an angle of $5^{\circ}$ between the incident ray and the normal to the blazed facet surface. A detector slit with size of $0.5 \mathrm{~mm}$ provided an angular resolution of $0.12^{\circ}$. Detector scans over the diffraction angle were performed at wavelengths between $12.8 \mathrm{~nm}$ and $14.7 \mathrm{~nm}$ with an increment of $0.05-0.1 \mathrm{~nm}$. The data were normalized to the direct beam intensity measured over the wavelength range.

A cross-sectioned grating sample was investigated with TEM in order to reveal the structure of the multilayer stack. TEM imaging was performed with a $200 \mathrm{kV}$ instrument. The details of cross-section sample preparation performed with a double column FIB instrument can be found elsewhere. ${ }^{8,9}$

\section{RESULTS AND DISCUSSION}

\subsection{Grating groove profile before and after multilayer deposition}

The perfection of the grating groove profile is one of the most important parameters for blazed gratings. It requires thorough characterization in order to estimate grating quality and predict grating efficiency. An SEM image of a silicon grating fabricated by wet anisotropic etch is shown in Fig. 2a. A grating groove consists of a 6-degree tilted blaze facet and a 64-degree anti-blaze facet. The grooves also have 20-25 nm wide nubs left behind after the lithography/etch step. The subsequent nub removal process results in a triangle-like shape of the grating grooves (Fig. 2b), though some residual bumps are still visible in the upper vertices of the grooves.

A high-resolution TEM image of the grating gives more details of groove shape in the vicinity of the anti-blaze facets (Fig. 3). The surface of the silicon grating is coated with a $1 \mathrm{~nm}$ thick layer of an amorphous silicon oxide which is a product of chemical oxidation of the silicon with Piranha solution. The self-limiting nature of silicon oxide growth provides high uniformity of the layer thickness. The uniformity of the oxidation and high selectivity of the oxide etch with HF provide gentle isotropic removal of the material without significant roughening of the surface. The measured oxide thickness value is consistent with the number of the cycles required for nub removal. Indeed, growth of $1 \mathrm{~nm}$ thick oxide layer consumes roughly $0.5 \mathrm{~nm}$ of the silicon; therefore about 20-25 cycles are needed to remove a 20-25 nm wide nub taking into account that material removal occurs from both sidewalls of the nubs.

The anti-blaze facets have the same slope angle of 64 degrees as directly after the $\mathrm{KOH}$ etch. The angle is determined by the angle between the blazed and anti-blazed facets, which is $109.47^{\circ}$ for the miscut towards the [112] direction, and the blaze angle of 6 degrees. Thus, the nub removal process does not affect the slope of anti-blaze facets formed by $\mathrm{KOH}$ etch.

The height of the bumps, which are residual nubs, does not exceed a few nanometers. An increase in the number of the cycles can provide complete removal of the nubs, however it is not necessary since these minor imperfections do not make any difference as will be shown below. A larger number of the cycles is not desirable because it would result in an increase of another imperfection of the groove. This is a rounding at the foot of the anti-blaze facets, well seen in Fig. 3. The rounding is a result of the oxidation-etch process which removes material in an isotropic manner. After KOH etch of a groove, schematically shown with a dotted line in Fig. 3, it has a sharp corner between blaze and anti-blaze facets. The isotropic removal process results in a rounded corner with a radius equal to the thickness of the removed layer. Thus, the rounding is an inevitable consequence of the nub removal procedure, and cannot be avoided completely. It can be reduced by through optimization of $\mathrm{KOH}$ etch time in order to obtain a minimum width of the nubs. In this case a smaller number of the cycles would result in smaller rounding. Another way to mitigate the rounding problem is use of silicon crystals with a miscut towards the $[11 \overline{2}]$ direction. This would result in a negative slope of anti-blaze facets, ${ }^{10}$ and at least a part of the rounding can be hidden behind the facets.

Although TEM images provide the most precise and reliable information on grating groove profile, the technique cannot be used for grating characterization on a regular basis because of the high cost of the TEM investigation and destructive character of the method. We used the AFM technique for routine surface roughness measurements and groove profile characterization. This non-destructive, inexpensive and fast technique provides quantitative information on surface relief 

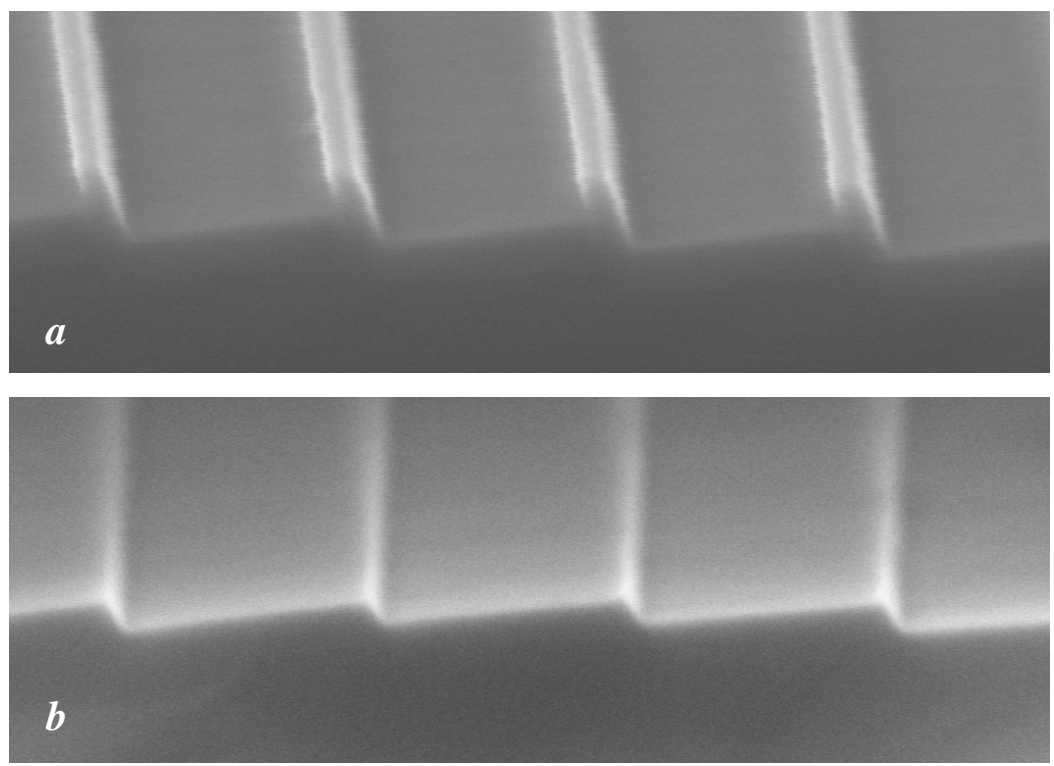

Figure 2. SEM images of the silicon blazed grating after anisotropic $\mathrm{KOH}$ etch (a) and after nub removal (b).

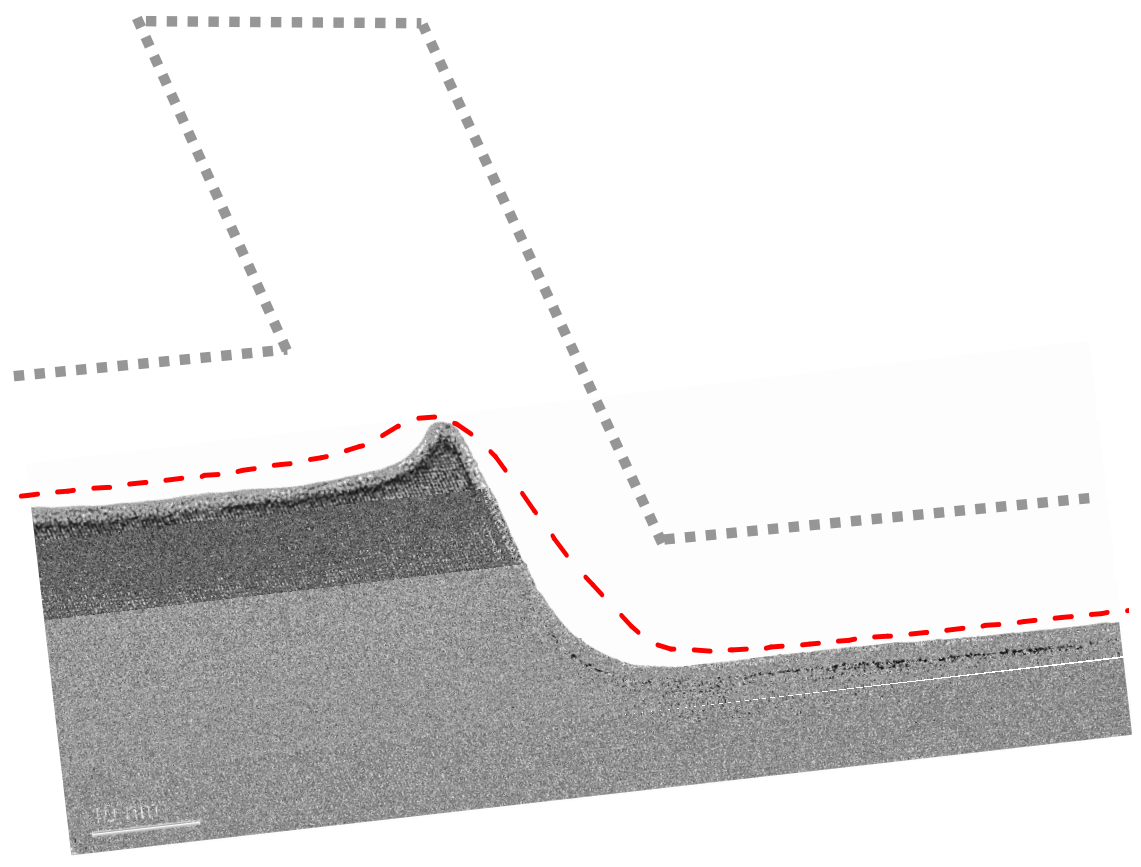

Figure 3. TEM image of the silicon blazed grating after nub remova1. Dotted line depicts a grating surface before nub removal. Dashed curve is a typical AFM profile.

and allows fast collection of datasets large enough for obtaining average groove profiles with good statistics for efficiency simulations. However, one should be concerned about the reliability of the AFM data since they affected by the AFM tip shape. A typical AFM trace obtained with a new tip with nominal radius of $7 \mathrm{~nm}$ is shown with a dash line in Fig. 3. One can see that small and high aspect ratio features of the sawtooth substrate appeared to be smoothed on the AFM profile. The bump looks much wider and the slope of the anti-blaze facet is not recorded perfectly. This is because the radius of the bump in Fig. 3 is about $1 \mathrm{~nm}$, which is smaller than a radius of even super sharp AFM tips, and even 
super sharp tips commercially available ${ }^{11}$ cannot follow the bump surface precisely. Nevertheless, a new sharp tip provides reliable AFM data on the blazed facet surface, and minimal distortions of the grating groove in the anti-blaze facet area should not affect much the efficiency of the blazed order. Average groove profiles measured with the AFM before and after the multilayer deposition as well as an ideal triangular profile shaped with the (111) planes of the silicon lattice are shown in Fig. 4. The profile of the silicon blazed grating (dotted line) is pretty close to the ideal one (dashed line), but has undergone significant smoothing after the multilayer deposition (solid line). In the next paragraph we will estimate the impact of the smoothing on grating performance with diffraction efficiency simulations.

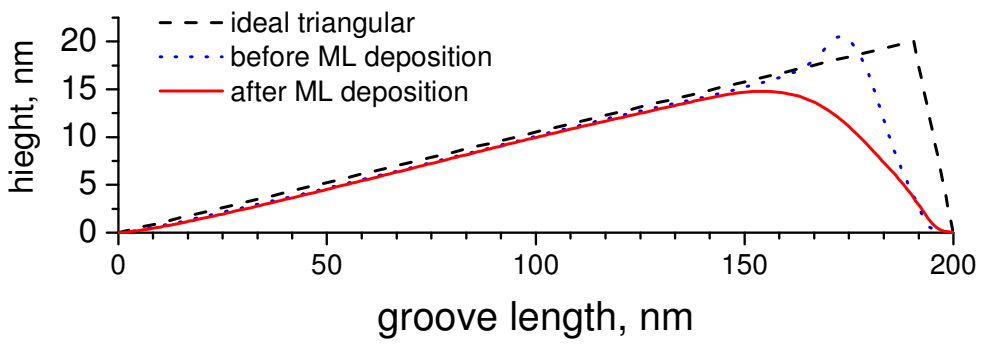

Figure 4. AFM-measured groove profiles of the blazed grating before (dotted curve) and after (solid curve) multilayer deposition. The dashed curve shows an ideal triangular profile.

\subsection{Measurements and simulation of diffraction efficiency}

Diffraction measurements and simulated diffraction patterns from the grating coated with the $\mathrm{Mo} / \mathrm{Si}$ multilayer at a wavelength of $13.6 \mathrm{~nm}$ are shown in Fig. 5, and dependence of the $3^{\text {rd }}$ diffraction order efficiency on wavelength is shown in Fig. 6. The grating demonstrated an absolute efficiency of $37.6 \%$ in the $3^{\text {rd }}$ diffraction order. To date this is the highest efficiency achieved for such a dense diffraction grating at EUV wavelengths.

In order to estimate the impact of the groove profile on grating performance, simulations of diffraction efficiency were performed for three different grating groove profiles shown earlier in Fig. 4. The model multilayer stacks used for simulations are shown on the right in Fig. 5. All the layers inside each stack were assumed to have the same profile, i.e. no changes of groove profile through the multilayer stack were considered. The multilayer stack models ignore a finite interface width and assume ideally sharp interfaces. In order to take into account a realistic interface width of $0.75 \mathrm{~nm}$ for the multilayer interfaces, the obtained efficiency values were reduced by a factor equal to 0.88 for the resonant wavelength of $13.6 \mathrm{~nm}$. The factor was calculated as a ratio of reflectance of an ideal Mo/Si multilayer to the reflectance of the same multilayer with $0.75 \mathrm{~nm}$ wide interfaces ${ }^{12}$ in the wavelength range under consideration.

The simulation results obtained for the grating with the smoothed profile demonstrate the best match of efficiency of the blazed order to the experimental data. This seems reasonable because the upper layers have the largest contribution to multilayer reflectance and grating efficiency, and adequate modeling of the shape of the upper part of the multilayer stack is most important. There is some quantitative discrepancy between experiment and simulation for efficiency of the non-blaze orders because of the simplicity of the model used. A more complicated model would take into account the real structure of the multilayer stack in order to obtain a better quantitative match to the simulated efficiency of all the orders in the experimental data.

The simulations show clearly the impact of groove profile distortions on the grating efficiency. An ideal triangular groove concentrates almost all diffracted energy in one blaze order, and provides the highest diffraction efficiency. Any deviation from the ideal triangular shape results in reduction of the blaze order efficiency and dissipation of a significant part of the energy, which partially goes into non-blaze orders. Since smoothing of the groove profile with the multilayer has a negative impact on grating efficiency, the understanding of multilayer growth is very important for further progress in grating quality. This is a relatively new and poorly investigated problem for multilayer deposition technology which deals usually with flat or slightly curved substrates. To our knowledge, multilayer growth on sawtooth substrates has never been published before. In the next section we present our first results on TEM examination of the real structure of the multilayer stack, which gives an insight into multilayer growth behavior. 

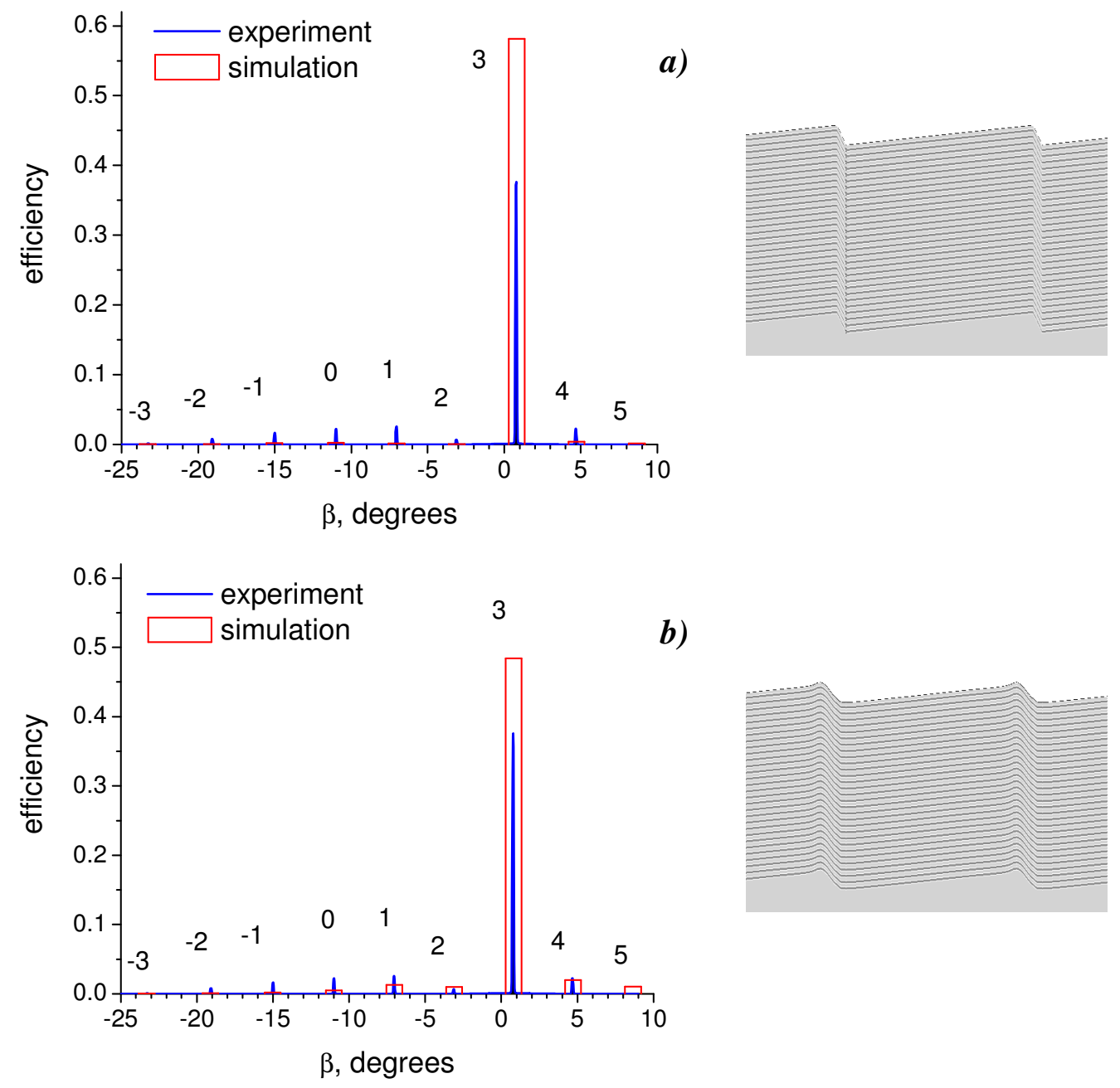

b)
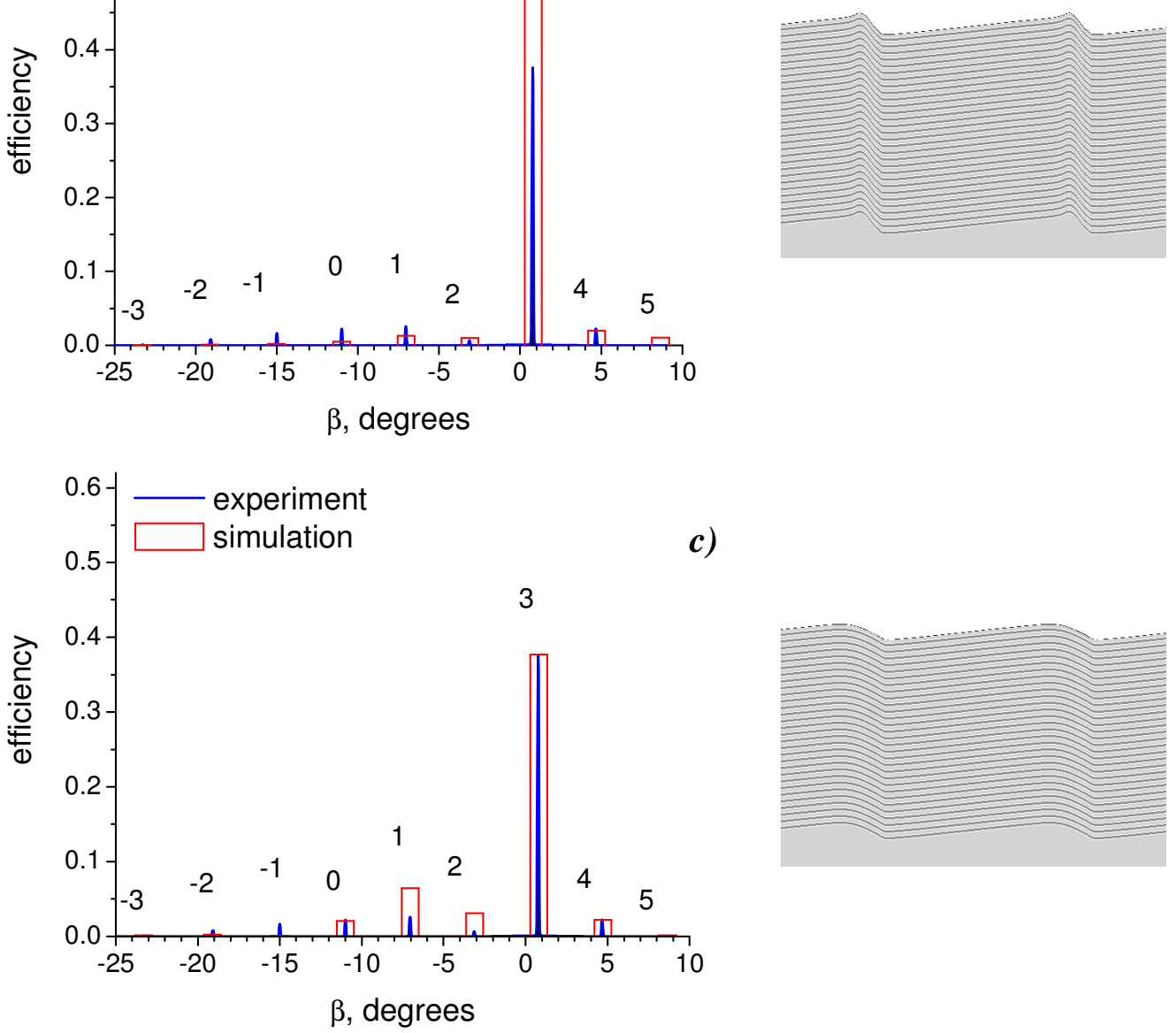

Figure 5. Experimental (solid line) and simulated (bars) diffraction obtained from the Mo/Si coated blazed grating with a period of $200 \mathrm{~nm}$ at the wavelength of $13.6 \mathrm{~nm}$. The measurements were performed at the fixed incident angle, $\alpha$, of $11^{\circ}$ by a detector scan over the diffraction angle, $\beta$. The simulations were performed for the ideal triangle groove profile (a), and the AFM-measured profiles before (b) and after (c) multilayer deposition. The multilayer stack models used for simulations are shown on the right. 


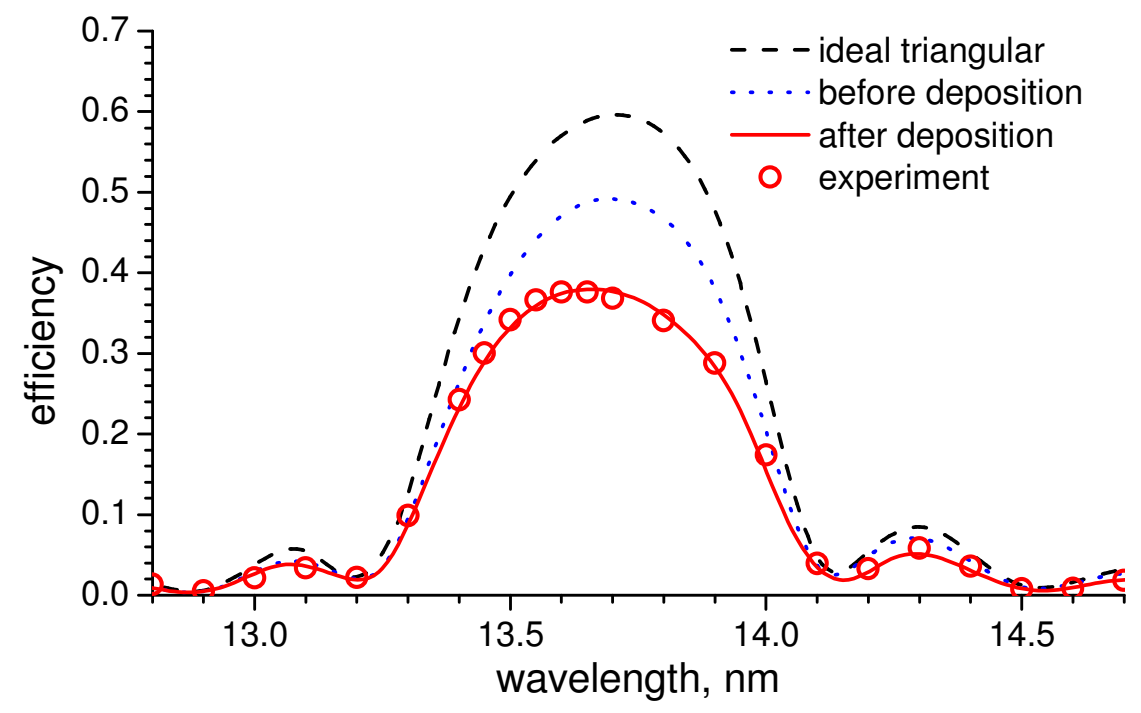

Figure 6. Dependence of diffraction efficiency of the $3^{\text {rd }}$ diffraction order of a Mo/Si coated blazed grating with a period of $200 \mathrm{~nm}$ on wavelength. The circles show experimental data, and the curves show simulated efficiency calculated for ideal triangle groove profile (dashed curve), and the AFM-measured profiles before (dotted curve) and after (solid line) multilayer deposition.

\subsection{TEM examination of multilayer stack structure.}

Transmission electron microscopy reveals the internal structure of the multilayer stack in detail, and gives insight into the multilayer growth behavior for a sawtooth substrate. A cross-sectional TEM image of the Mo/Si coated grating is shown in Fig. 7. The molybdenum layers look dark because of high absorption of electrons, while the silicon ones are light. The TEM image shows a complicated evolution of the structure of the multilayer stack in the course of the deposition, which results in distortion of the groove profile of the grating. The high frequency features of the substrate relief (shown by the numbers in Fig. 7) undergo the most dramatic modifications. They change beyond recognition or disappear completely and get replaced with new defects. The slope angle of the anti-blaze facets reduces significantly after the deposition. There is no evidence of a bump (1) in the upper top surface of the multilayer, and on the contrary, the top vertex of the originally triangular groove is severely smoothed and rounded (3). The rounding (2) at the foot of the anti-blazed facet of the silicon substrate is replaced by a trough (4) on the top of the multilayer stack. As a result of these distortions, a substantial portion of the multilayer stack is perturbed (5). There are areas where distortions are so large that the multilayer structure collapses completely (6).

It is known that multilayer structure is a result of competition and interplay of shadowing and smoothing processes taking place on the surface of a growing film. ${ }^{13,14}$ The random nature of deposition causes stochastic fluctuations of the local deposition rate, resulting in surface roughening by formation of high frequency surface defects like bumps and pits. ${ }^{15}$ These high frequency features can cause shadowing of incoming atom flux. Reduction of the deposition rate in shadowed areas enhances the shadowing effect and the growth of the defects. However, surface irregularities can be smoothed out via surface diffusion of the atoms. The curvature of the surface in the vicinity of a high frequency defect gives rise to a gradient of chemical potential which is a driving force for diffusion flux..$^{16}$ If atom surface mobility is high enough, the flux provides an effective smoothing of surface irregularities by a mass transfer from concave surface areas to convex ones. Otherwise, shadowing dominates over the smoothing and a columnar structure of the multilayer stack appears. ${ }^{13,17,18}$ These issues are well studied for the multilayers deposited on flat substrates. However one can expect much more complicated growth behavior for corrugated surfaces, because a sawtooth substrate has its own high frequency features which will introduce additional perturbations in the deposition process.

Fig. 8a shows the propagation and transformation of the substrate relief through the multilayer stack in detail. The propagation direction is perpendicular to the layers and shown with a dashed line drawn via the bump in Fig. 8a. Due to the very large difference in the surface slope of the groove facets, multilayer growth occurs in very different regimes at 


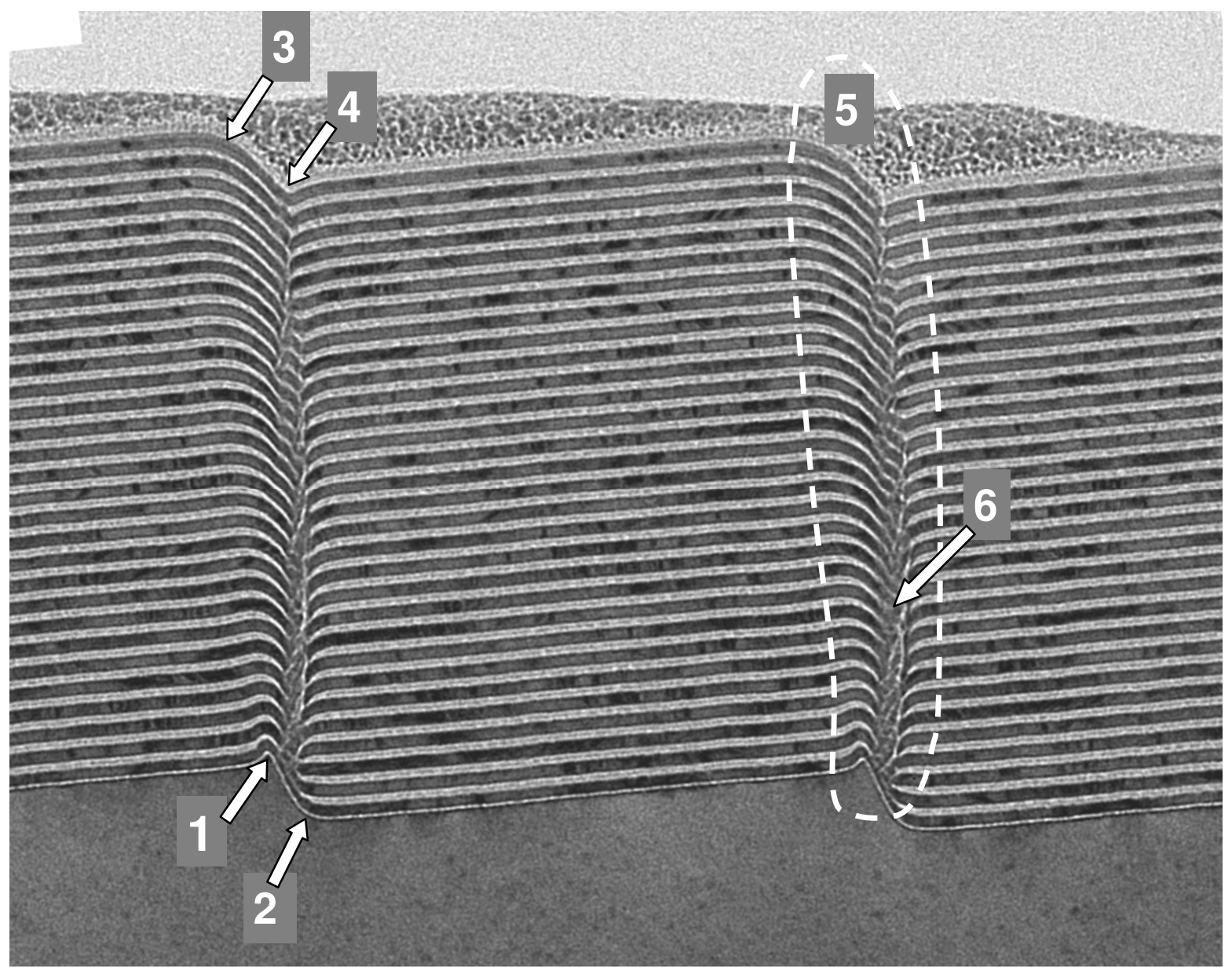

Figure 7. TEM image of a cross-section of a blazed grating, coated with $30 \mathrm{Mo} / \mathrm{Si}$ bi-layers. The silicon grating substrate has 6-degree blaze facet and 64-degree anti-blaze facet. There are small bumps (1) in the upper part of the grooves and a rounding (2) of the bottom junction of the facets. After the deposition the grating profile changes significantly, there are rounded vertices (3) and a trough (4) on the top surface of the ML. A significant volume of the multilayer stack is perturbed (5), and areas with collapsed multilayer structure (6) are observed.

the blaze facet side (to the left of the dashed line) and anti-blaze facet side (to the right of the line). The difference becomes obvious when we cut the image into two parts along the dashed line, and then replace the right part by a mirror copy of the left one and vice versa as shown in Fig. 8b and Fig. 8c. It is well visible that the multilayer grows in a smoothing regime at the blazed facet side (Fig. 8b). The multilayer structure here is essentially a record of the smoothing process. Note, that thickness of silicon layers on the top of the bump is much smaller compared to the layers resting on a flat surface of the blazed facet. Since the average deposition rate was the same over the substrate area, the nonuniformity of the thickness of silicon layers indicates a diffusion mass transfer of silicon atoms away from the highly convex surface of the top of the bump towards concave areas at the foot of the bump. The foot of the bump might suffer from some shadowing because of the wide cone of the flux of arriving atoms. The shadowing was obviously overcome in this case by the surface diffusion which provides effective smoothing of the bump.

The silicon atom mobility can be roughly estimated from the smoothing data by the formula $\boldsymbol{x}^{2}=2 \boldsymbol{D} \tau$, where $\boldsymbol{x}$ is a diffusion length, $\boldsymbol{D}$ is a diffusion coefficient, and $\tau$ is a time of diffusion. For a reasonable diffusion length of a few nanometers and time of diffusion of $0.5 \mathrm{sec}$ (which is approximately the time of one monolayer growth) the surface diffusivity of the silicon is roughly $10^{-16} \mathrm{~m}^{2} / \mathrm{sec}$. This value is rather high for ambient temperature, and probably reflects the relatively high energy of the magnetron sputtered atoms and some assisting of the atom motion by backscattered Ar neutrals. ${ }^{19}$ As opposed to silicon layers, the thickness of molybdenum layers doesn't change much in vicinity of the 

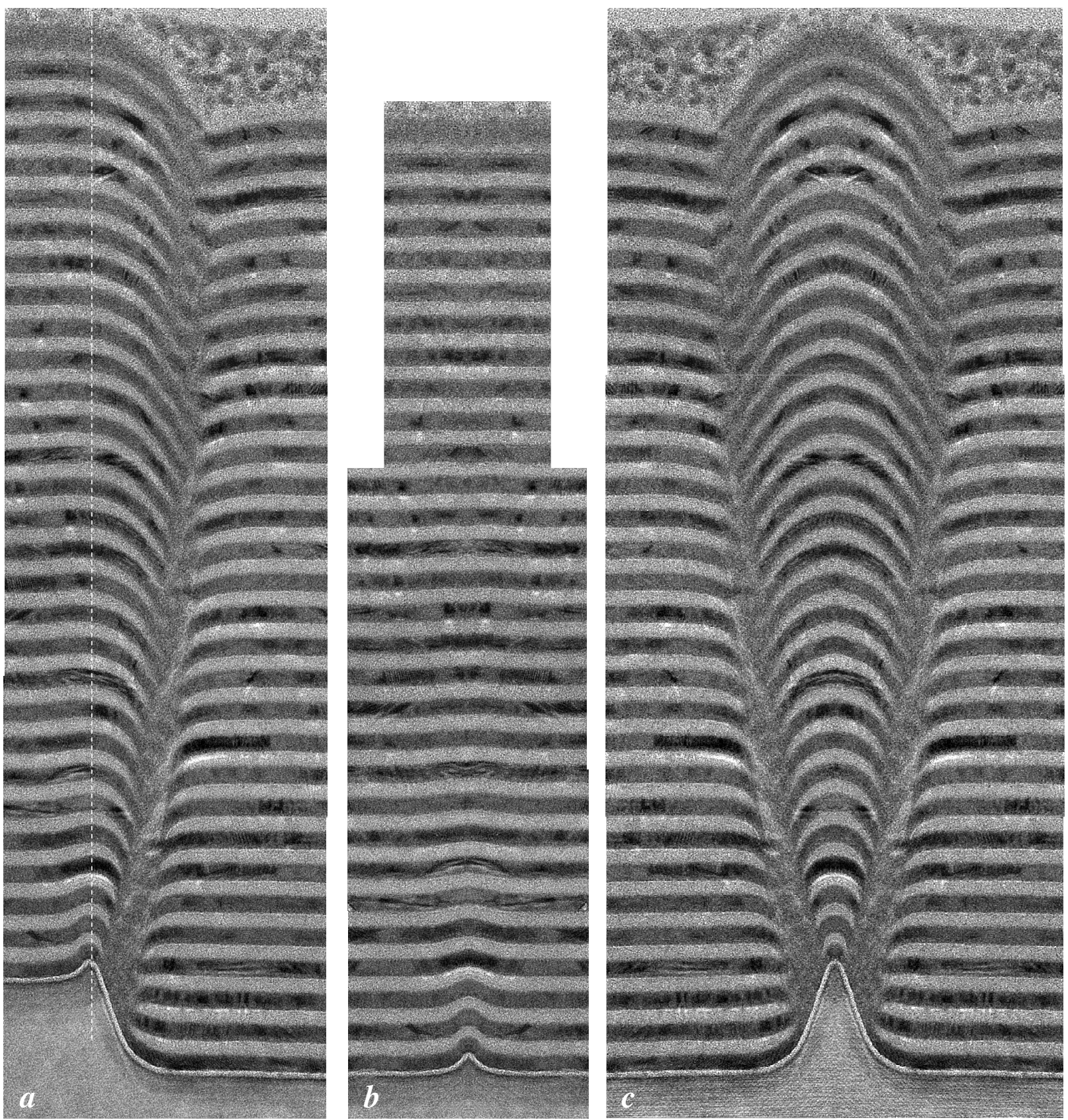

Figure 8. Structure of the multilayer stack in vicinity of an anti-blazed facet: (a) a real cross-section TEM image; (b) a virtual TEM image illustrating smoothing regime of the multilayer growth in the area to the left from the dashed line shown in (a); (c) a virtual TEM image illustrating shadowing and column-like regime of the multilayer growth to the right of the dashed line in (a). See more comments in the text.

bump. This is due to the much smaller mobility of molybdenum surface atoms. Indeed relatively high mobility of silicon atoms is well known for the processes of $\mathrm{Mo} / \mathrm{Si}$ multilayer growth and used for substrate defect planarization. ${ }^{20-22}$

At the anti-blaze facet side, multilayer growth occurs in a much more complicated manner. In the beginning of the deposition shadowing predominates over diffusion smoothing, and a pronounced column growth structure appears (Fig. 8c). One can follow the structural changes layer by layer. The first deposited layer of molybdenum seems to be continuous and have uniform thickness measured in the growth direction. This indicates that there was no shadowing in the beginning of the deposition, and corresponds to the expectation that the 10-degree tilt of average atom flux direction 
(see Fig. 1) should rule out any shadowing. However during the deposition of the next layer, which is silicon one, a sufficient diffusion redistribution of the material occurs. Thickness of the silicon layer on the top of the bump reduces, at the same time the upper part of the bump becomes slightly wider. This tiny change of the bump shape results in significant shadowing because of a very oblique deposition angle for the anti-blaze facet surface; in addition, the antiblaze facet surface appears to be deeply shadowed. Note that the surface diffusion results in smoothing of a small bump (Fig. 8b), but triggered shadowing in the case of a large bump (Fig. 8c).

The area at the foot of the anti-blaze facet also suffered from the shadowing, as one can judge from the changes of the multilayer stack here. In approaching the anti-blaze facet, the layers become thinner because of growing shadowing. The thickness change affects the shape of the layers. At first, only a few layers follow the substrate rounding and are bent up. Then the bend decreases for each subsequent layer, and bending up is soon replaced with bending down. In this way, the original rounding of the substrate transforms into a trough at the foot of the anti-blaze facets. The trough probably gives rise to diffusion of atoms towards the shadowed area in order to fill the trough. The difference in surface mobility for silicon and molybdenum atoms can explain the relatively large changes of the thickness of the deformed silicon layers compared to the molybdenum ones.

Shadowing has its largest impact on the thickness of the layers deposited on the anti-blazed facets. Note that even with no shadowing the layer here would be approximately twice as thin because of a cosine factor for deposition on a tilted surface. A cumulative effect of the geometry and the shadow makes the silicon and molybdenum layers so thin that they intermix completely and the layer structure collapses. An amorphous material which is probably a molybdenum silicide is observed instead of silicon and molybdenum layers. The collapsed area looks to be filled with this material. This however can be an artifact of the cross-section sample preparation technique which involves intensive sputtering with an ion beam followed by re-deposition of the sputtered material. One would assume void formation in shadowed areas, which is typical for the column growth regime. ${ }^{18}$ We observed cracks in between the grooves in the SEM images of the cleaved gratings. ${ }^{1}$ The cracks show a lack of strength of the joints of the multilayer stacks resting on adjacent grooves, and supports the possibility of the presence of voids and discontinuity of the layers in the collapsed area.

The bump in Fig. 8c becomes wider with total thickness of the coating, and a wider area of the multilayer stack is affected by perturbations. At the same time, the originally sharp bump transforms into a wide rounded hill with gentle slopes. A smoothed profile causes weaker shadowing, and a transition to a smoothing regime of the multilayer growth occurs gradually. As a result, the collapsed area fills, and the amplitude of layer distortion decreases, and the troughs become smaller. Eventually the layer discontinuity is replaced by continuous stitching of the layers at the bottom of the troughs (pointed to by the number 4 in Fig. 7). One can expect further smoothing of the groove profile for larger coating thicknesses, and formation at some point of a sine-like groove profile.

The multilayer growth scenario described above is from a preliminary analysis of the multilayer stack structure. It helps to understand the main transformation of the profile of the grating, based on simple shadowing/smoothing considerations. However, details of the growth can also depend on other processes which have been not taken into account. Impact of re-sputtering of the materials, dependence of sticking factors on the angle of deposition etc. cannot be analyzed in this simple way. Comprehensive analysis which would provide a quantitative description of the multilayer growth will be a subject of future work.

Since distortions of the multilayer stack have a negative impact on grating efficiency, minimization of the layer perturbations seems to be a most important challenge. The multilayer growth regime strongly depends in particular on the shape of a surface feature on a substrate. Relatively small bumps are easily smoothed with the multilayer (Fig. 8b), while the larger features with steep sidewalls provoke significant shadowing under the same deposition condition (Fig. 8c). This should be taken into account in grating design in order to minimize the multilayer stack warp. For example, reduction of a grating period or a blaze angle would reduce the height of the anti-blazed facets, and one can expect mitigation of shadowing and hopefully perturbations. This approach requires an additional investigation though.

While the final groove profile depends strongly on the height and slope angle of the anti-blaze facets, it seems to be fairly independent of the fine details of the substrate. The groove imperfections, like the bumps and the rounding are not translated through the multilayer stack; they decay after a deposition of a few layers. That means that perfection of the groove profile of a sawtooth substrate is not relevant so much for the deposition procedure used in this work. One would expect a pretty similar smoothing of the grooves by the multilayer for the ideal triangular groove profile of the substrate. Grating performance seems to be limited by deposition challenges rather than substrate quality. Hence, the deposition requires additional improvement in order to take control of both the shadowing and smoothing processes. 
Shadowing obviously depends on the divergence of the incident atomic flux, and so it can be reduced significantly by collimation of the flux and choice of an optimal angle of deposition. It seems to be relatively easily controlled by an appropriate hardware development. The smoothing depends first of all on intrinsic properties of the deposited material, namely on surface diffusion coefficients. It is probably not so easy controlled because of a limited choice of the materials suitable for the EUV and soft x-ray multilayers.

The surface mobility can be varied in a wide range by tuning the energy of the deposited atoms, but very low mobility would result in high interface roughness. Finding a tradeoff between minimal profile smoothing and an acceptable level of interface roughness is one of the ways forward in deposition optimization.

Another possible way to reduce the smoothing is optimization of the $\Gamma$-ratio of a multilayer, i.e. the metal layer thickness divided by the multilayer period. Thickness of the layer responsible for the smoothing can be reduced in order to mitigate a smoothing problem. This approach does not worsen the interfaces, but is limited by the reasonable range of the $\Gamma$-ratio since the reflectance of the multilayer would suffer if the ratio is far from the optimal value.

All of these points mentioned above indicates that deposition of multilayers on such corrugated surface requires a specific optimization strategy involving the choice of multilayer materials, $\Gamma$-ratio of the multilayers, the angle of deposition, energy of atoms arriving to a substrate, flux divergence, etc., and can be quite different compared to the relatively simple case of multilayer deposition on a flat substrate. Numerical multilayer growth simulation taking into account the full set of deposition parameters including surface diffusion, sticking factors, re-sputtering etc. would be very helpful. A few examples of the simulation approaches developed for the different deposition techniques used for microfabrication purposes look promising and should be tried for multilayer growth simulations. ${ }^{23-25}$

\section{CONCLUSIONS}

A highly reliable process of blazed grating fabrication and comprehensive grating characterization was developed. The high quality of the silicon sawtooth substrates provided a record efficiency for a multilayer coated blazed grating with a high groove density and dispersion. The highest diffraction efficiency was achieved for the $3^{\text {rd }}$ diffraction order of the 5000 lines $/ \mathrm{mm}$ grating at the wavelength of $13.6 \mathrm{~nm}$. Careful characterization of the grating groove profile combined with diffraction efficiency simulation provides prediction of the grating performance and quantitative estimation of the impact of groove profile imperfection on grating performance. It was found that smoothing of the grating groove profile by the multilayer results in significant efficiency losses.

The structure of the multilayer deposited on the sawtooth substrate was studied with cross-sectional TEM for the first time. It was found that changes of the groove profile in the course of the deposition are caused by shadowing and smoothing processes which result in significant changes in the groove profile in the vicinity of the anti-blaze facets. The original imperfections of the groove profile do not penetrate through the multilayer stack and do not have a significant impact on the final groove profile. The grating performance is limited by the deposition procedure applied rather than the substrate imperfections. The deposition requires significant improvements in order to obtain control over the shadowing and smoothing processes in effect in the course of the multilayer growth on the highly corrugated surface of a blazed grating.

\section{ACKNOWLEDGEMENTS}

The authors are very thankful to Wayne McKinney and Sunling Yang for useful discussions and invaluable assistance in the grating efficiency measurements.

The Advanced Light Source is supported by the Director, Office of Science, Office of Basic Energy Sciences, Material Science Division, of the U.S. Department of Energy under Contract No. DE-AC02-05CH11231 at Lawrence Berkeley National Laboratory. 


\section{DISCLAIMER}

This document was prepared as an account of work sponsored by the United States Government. While this document is believed to contain correct information, neither the United States Government nor any agency thereof, nor The Regents of the University of California, nor any of their employees, makes any warranty, express or implied, or assumes any legal responsibility for the accuracy, completeness, or usefulness of any information, apparatus, product, or process disclosed, or represents that its use would not infringe privately owned rights. Reference herein to any specific commercial product, process, or service by its trade name, trademark, manufacturer, or otherwise, does not necessarily constitute or imply its endorsement, recommendation, or favoring by the United States Government or any agency thereof, or The Regents of the University of California. The views and opinions of authors expressed herein do not necessarily state or reflect those of the United States Government or any agency thereof or The Regents of the University of California.

\section{REFERENCES}

[1] Voronov, D. L., Anderson, E. H., Cambie, R., Salmassi, F., Gullikson, E. M., Yashchuk, V. V., Padmore, H. A., Ahn, M., Chang, Ch.-H., Heilmann, R. K., Schattenburg, M. L., "5000 groove/mm multilayer-coated blazed grating with $33 \%$ efficiency in the $3^{\text {rd }}$ order in the EUV wavelength range," Proc. SPIE 7448, 74480J (2009).

[2] Workshop on "Soft X-Ray Science in the Next Millennium: The Future of Photon-In/Photon-Out Experiments." (Pikeville, Tennessee March 15-18, 2000), http://www.phys.utk.edu/WPWebSite/ewp_workshop_XRay_Report.pdf

[3] Kowalski, M. P., Barbee, Jr., T. W., Hunter W. R., "Replication of a holographic ion-etched spherical blazed grating for use at extreme-ultraviolet wavelengths: efficiency," Appl. Opt. 45(2), 322-334 (2006).

[4] http://henke.lbl.gov/multilayer/survey.html

[5] http://www-cxro.lbl.gov/laboratories/coatings

[6] http://www.pcgrate.com/

[7] http://www-cxro.lbl.gov/beamlines/6.3.2

[8] http://www.eaglabs.com/techniques/analytical_techniques/fib.php

[9] Yashchuk, V. V., Conley, R., Anderson, E. H., Barber, S. K., Bouet, N., Voronov, D. L., "Development of a binary pseudo-random multilayer test sample for MTF measurements with electron microscopes: FIB sample preparation and TEM measurements," LSBL report.

[10] Philippe, P., Valette, S., Mata Mendez, O. and Maystre, D., "Wavelength demultiplexer: using echelette gratings on silicon substrate, " Appl. Opt. 24(7), 1006-1011 (1985).

[11] https://www.veecoprobes.com/

[12] http://henke.lbl.gov/optical_constants/multi2.html

[13] Stearns D. G., Rosen, R. S., Vernon, S. P., "Fabrication of high-reflectance Mo-Si multilayer mirrors by planar magnetron sputtering," J. Vac. Sci. Technol. A 9 (5), 2662-2669 (1991).

[14] Spiller, E., Baker, S., Parra, E., Tarrio, C., "Smoothing of Mirror Substrates by Thin-Film Deposition," Proc. SPIE 3767, 143-153 (1999).

[15] Spiller, E. [Soft X-ray Optics], Bellingham, Washington (USA): SPIE Opt. Eng. Press, 279 (1994).

[16] Stearns, D. G., Gaines, D. P., Sweeney, D. W., Gullikson, E. M., "Nonspecular x-ray scattering in a multilayercoated imaging system," J. Appl. Phys., 84(2), 1003-1028 (1998).

[17] Vernon S. P., Stearns D. G., Rosen R. S. "Ion-assisted sputter deposition of molybdenum-silicon multilayers," Appl. Opt., 32(34), 6969-6974 (1993).

[18] Niibe, M., Hayashida, M., Iizuka, T., Miyake, A., Watanabe, Y., Takahashi, R., Fukuda, Y., "Suppression of columnar-structure formation in Mo-Si layered synthetic microstructures," Proc. SPIE 1343, 2-13 (1990).

[19] Hoffman, D. W., Thornton, J. A., "Compresive stress and inert gas in Mo films sputtered from a cylindrical-post mafnetron with Ne, Ar, Kr, and Xe," J. Vac. Sci. Technol. 17, 380-383 (1980).

[20] Mirkarimi, P. B., Spiller, E., Baker, S. L., Robinson, J. C., Stearns, D. G., Liddle, J. A., Salmassi, F., Liang, T., Stivers, A. R., "Advancing the ion beam thin film planarization process for the smoothing of substrate particles," Microelectron. Eng. 77, 369-381 (2005). 
[21] Mirkarimi, P. B., Spiller, E., Baker, S. L., Stearns, D. G., Robinson, J. C., Olynick, D. L., Salmassi, F., Liddle, J. A., Liang, T., Stivers, A. R., "A silicon-based sequential coat-and-etch process to fabricate nearly perfect substrate surfaces," J. Nanosci. and Nanotechnol. 6, 28-35 (2006).

[22] Mirkarimi, P. B., Spiller, E. A., Stearns, D. G., Sperry, V., Baker, Sh. L., "An ion-assisted Mo-Si deposition process for planarizing reticle substrates for extreme ultraviolet lithography," IEEE J. Quantum Electron. 37(12), 1514-1516 (2001).

[23] Kwon, U. H., Choi, S. H., Park, Y. H., Lee, W. J., "Multi-scale simulation of plasma generation and film deposition in a circular type DC magnetron sputtering system," Thin Solid Films 475, 17- 23 (2005).

[24] Cale, T. S., Jain, M. K., Taylor, D. S., Duffin, R. L., Tracy, C. J., "Model for surface diffusion of aluminum (1.5\%) copper during sputter deposition," J. Vac. Sci. Technol. B 11(2), 311-318 (1993).

[25] Cale, T. S., Merchant, T. P., Borucki, L. J., Labun, A. H., "Topography simulation for the virtual wafer fab," Thin Solid Films 365, 152-175 (2000). 